\title{
Carnets
}

Revue électronique d'études françaises de l'APEF

Première Série - $1 \mid 2009$

La mer... dans tous ses états

\section{La Mer Méditerranée. Lieu et non-lieu dans N’zid et Mes Hommes de Malika Mokeddem}

\section{Anne Aubry}

\section{OpenEdition}

Journals

Édition électronique

URL : http://journals.openedition.org/carnets/2760

DOI : 10.4000/carnets. 2760

ISSN : 1646-7698

Éditeur

APEF

Édition imprimée

Pagination : 17-31

\section{Référence électronique}

Anne Aubry, «La Mer Méditerranée. Lieu et non-lieu dans N'zid et Mes Hommes de Malika Mokeddem », Carnets [En ligne], Première Série - 1 | 2009, mis en ligne le 13 juin 2018, consulté le 20 avril 2019. URL : http://journals.openedition.org/carnets/2760 ; DOI : 10.4000/carnets.2760

\section{(c) (1) \&)}

Carnets est mis à disposition selon les termes de la licence Creative Commons - Atribution - Pas d'utilisation commerciale 4.0 International. 


\section{LA MER MEDITERRANEE \\ Lieu et non-lieu dans N'zid et Mes Hommes de Malika Mokeddem}

ANNE AUBRY

Universidad Pablo de Olavide

acaubx@upo.es

\section{Résumé}

Pour Malika Mokeddem, la mer est un élément fondamental de sa représentation du monde. La Méditerranée est en effet la frontière entre ses deux champs d'action fictionnels et autobiographiques que sont l'Algérie et la France. De cette double culture et de cette double appartenance, de ce double arrachement aussi, Malika Mokeddem tire la substance d'une création littéraire caractérisée par une tension entre le désert et la Méditerranée.

Ces espaces sont simultanément des lieux et des "non-lieux", ce sont des créations littéraires, des projections d'aspirations et de luttes intérieures, des allégories de batailles gagnées et de victoires sur la peur, les tabous et les poids des conventions.

\section{Abstract}

For Malika Mokeddem, the sea is a fundamental element in her representation of the world. The Mediterranean Sea is indeed the border between her two fictional and autobiographical fields of action: Algeria and France. From this double culture, this double belonging and equally this double rending, Malika Mokeddem moulds the substance of a literary creation characterized by tension between the desert and the Mediterranean.

These zones are simultaneously places and "no-places"; they are literary creations, projections of aspirations and of internal conflicts, allegories of battles won and victories over fear, taboos and the weights of convention.

Mots-clés: Malika Mokeddem, Littérature francophone du Maghreb, Désert, Intertextualité, Transtextualité, Identité

Keywords: Malika Mokeddem, French-speaking literature of the Maghreb, Desert, Intertextuality, Transtextuality, Identity 
Pour Malika Mokeddem, la mer est un élément fondamental de sa représentation du monde. La Méditerranée est en effet la frontière entre ses deux champs d'action - fictionnels et autobiographiques - que sont l'Algérie et la France. De cette double culture et de cette double appartenance, de ce double arrachement aussi, Malika Mokeddem tire la substance d'une création littéraire caractérisée par une tension entre le désert et la Mer Méditerranée.

Le désert, pour Malika Mokeddem, a une forte charge cosmique et métaphysique, mais avant tout, il s'agit pour elle d'un lieu d'identification. C'est en effet l'espace de son enfance et de son adolescence, le lieu du nomadisme de ses aïeux. Pourtant, elle perçoit aussi le désert comme une prison, comme une claustration et la mer apparaît alors comme une délivrance, une libération possible.

Nous observerons ainsi dans deux œuvres, l'une de fiction, N'zid (Mokeddem, 2001), l'autre de facture autobiographique, Mes Hommes (Mokeddem, 2006) le traitement de la thématique maritime perçue comme échappatoire, mais aussi comme lieu de contemplation et de sensibilité.

\section{Le désert dans Mes Hommes}

C'est l'élément le plus présent dans les cinq premiers romans de Malika Mokeddem: Les Hommes qui marchent (Mokeddem, 1990), Le Siècle des Sauterelles (Mokeddem, 1992), L'Interdite (Mokeddem, 1993), Des rêves et des assassins (Mokeddem, 1995), La nuit de la Lézarde (Mokeddem, 1998). Comme le souligne Najib Redouane, "un des traits essentiels du désert sur le plan de la création littéraire, c'est qu'il constitue le lieu identitaire de l'écrivaine" (Redouane, 2003: 23). En effet, l'œuvre Mes Hommes respecte particulièrement le "pacte autobiographique" de Philippe Lejeune que ce dernier oppose au "pacte de fiction".

Selon lui, l'autobiographie est un "récit rétrospectif en prose qu'une personne réelle fait de sa propre existence, lorsqu'elle met l'accent sur sa vie individuelle, en particulier sur I'histoire de sa personnalité." (Lejeune, 1975:14). C'est bien ce que promet Malika Mokeddem en écrivant Mes Hommes. Elle y expose sa lutte acharnée pour s'affranchir de son milieu, de sa famille où elle se sent niée et abaissée puisque sa condition de femme la condamne à l'enfermement et à la servitude. L'auteure, en dénonçant cette aliénation, établit un lien entre le désert et ce qu'elle a voulu fuir. En effet, le désert représente de manière emblématique le lieu de sa claustration et de sa domination.

Les mères ressassent à leurs filles, dès le plus jeune âge: "Il faut que tu aies honte. Tu dois avoir honte. Ne lève pas tes yeux sur les garçons. Sur les hommes. Baisse la tête. Dans la rue surtout. Ne te détourne pas. Si je te parle de honte, c'est que tu 
manques de pudeur...". A cette époque dans le désert, les filles s'inclinent, se ferment, se recroquevillent... (Mokeddem, 2006: 29)

Thérèse Michel-Mansour a analysé avec soin la figure du désert dans l'œuvre de Mokeddem et elle a relevé trois valeurs symboliques: premièrement, le désert est un espace métaphysique, car c'est le lieu de la marche. En se déplaçant, le corps atteint une valeur symbolique et parvient à une victoire sur l'immobilité. C'est un triomphe sur la mort qui nous enferme, nous réduit et nous cantonne à un seul lieu, celui de notre "repos éternel".

De plus, notre auteure établit un parallèle entre la marche et l'écriture, ces deux actions assurant une fonction thérapeutique. Pour Mokeddem, il faut "échapper", fuir l'enfermement, et marcher et écrire permettent de dépasser cette angoisse, de calmer la peur et de se sentir vivante. En marchant, en se déplaçant, en écrivant, en n'étant jamais "assignée à résidence", en sortant toujours de tout cadre, de toute structure, elle manifeste haut et fort son caractère indomptable. Par le même mouvement et simultanément, elle soigne les blessures passées, les souffrances endurées et l'énergie qu'elle a dû dépenser pour conquérir de haute lutte le droit de pouvoir "avancer".

Deuxièmement, le désert est un centre cosmique, très signifiant pour ceux qui savent s'y repérer. Troisièmement, le désert est le lieu où s'applique l'effort, l'application physique et métaphysique pour apercevoir l'Etre divin caché:

Ainsi, toujours est-il que l'œuvre de Mokeddem accorde à la symbolique du désert une importance insolite, dénomme ses multiples espaces. Espace métaphysique ou cosmique, le désert et ses immensités nous livrent un réel autre et nous invitent à partager les codes préexistants. À les chevaucher pour allier des dimensions ambivalentes. Cependant, le penchant de l'auteure à qualifier le désert d'univers cosmique où les repères nécessaires à l'orientation du nomade (la lumière des regards; la Présence qui veille) évoquent celui de la Présence de Dieu dans le sens ismaélien, nous semble particulièrement innovateur. Car c'est bien à travers cette expérience métaphysique que le nomade accède à une épuration de l'être, à une immatérialité de l'âme puisqu'il n'est plus qu'un rayon du firmament. Le nomade fait l'expérience du sacré dans le désert (Michel-Mansour, 2003: 182).

Pourtant, dans le texte qui nous intéresse, le désert apparaît essentiellement comme lieu d'enfermement, de claustration:

Là-bas, dans le désert, l'horizon n'était que l'ultime claustration. II symbolisait l'infranchissable de ma vie. L'insondable abîme qui me séparait du monde. De la liberté. Plus je grandissais, plus le vide du désert me serrait à la poitrine, à la gorge. A 
scruter ce néant immuable, ses paysages fossilisés qui cernaient notre pauvreté, la brutalité des traditions, j'avais parfois des crises de désespoir à en crever tant il me paraissait impossible que je puisse jamais décamper de là. Leur échapper (Mokeddem, 2006: 161).

Face à une telle violence, il est indispensable de trouver l'énergie, d'où qu'elle vienne, pour échapper à cette négation de l'être: "Toute une année scolaire. Une délivrance. [...] J'ai l'impression que le ciel du désert n'est plus un couvercle sur ma tête. Une chape qui me rive dans les exhalaisons de la maison. Je respire mieux. J'ai conscience d'avoir accédé à un champ de libertés qui bouscule tout..." (Mokeddem, 2001: 34).

Et le seul instrument qui permette de fuir est l'imagination, et particulièrement la lecture, antichambre de l'écriture.

\section{La face lumineuse du désert}

Cet espace devient tour à tour lieu d'inspiration et de création:

J'ai persisté à "peindre". Par moments. Par crises. Par transes. Le désert évidemment. Un désert dont les violences ont vite viré en abstractions. Une fureur, un déchirement de la couleur qui étaient d'abord des torsions physiques. [...] Comment assumer cet inconciliable: ce désert, ses brutalités imprimées sur ma rétine, dans ma sensibilité et mon inspiration à l'amour, à l'ailleurs? [...] Moi, l'attentive au sens des mots, celui de désert me résumait et me donnait envie de fuir (Mokeddem, 2006: 64$65)$.

La création revêt diverses formes, mais elle peut faire naître l'inspiration de l'écriture, c'est ainsi le cas de Tayeb, frère de Malika Mokeddem. Ce dernier a dû partir vivre aux Pays-Bas pour pouvoir se consacrer à la création, mais pour lui, le désert est un puissant stimulant pour l'écriture: “J'ai appris récemment, lors d'un voyage en Algérie, que Tayeb se rend de temps en temps au désert. Qu'il parcourt la contrée en solitaire. Qu'il projette de retourner vivre en Algérie" (Mokeddem, 2001: 199).

Dans ce texte autobiographique, les personnes, comme Tayeb, qui ont partagé son enfance et son adolescence sont aussi le prétexte à revenir à ce lieu d'ancrage. Ainsi, le photographe, celui qu'elle appelle "l'homme de mes images", Bellal, la retrouve à l'hôpital dans lequel elle exerce comme médecin alors qu'il doit se faire opérer:

A son retour, j'accompagne son chariot jusqu'au bloc, il me fixe avec anxiété et interroge: “Est-ce que la Barga te manque?". La Barga est la dune de mon enfance et 
de mon adolescence. II a posé cette question du ton des demandes essentielles. Celles qui s'imposent dans les moments critiques. "Oui, terriblement. C'était le tremplin de mes rêves". C'est la première fois que je reconnais ce manque (Mokeddem, 2006: 137).

Reconnaître le manque, c'est aussi apprivoiser le désir qui n'a pas été comblé, et rêver est une autre "voie royale" pour être au plus près de ses désirs et prendre conscience de la douleur. Le rêve final sur lequel se clôt pratiquement le récit est celui de l'accomplissement et de la réconciliation entre ces deux mondes: "Le prochain amour, il faudra l'amener sur la dune. Tant pis pour mon père s'il n'est pas d'accord! II ne le verra pas. Ça ne change rien!" (Mokeddem, 2001: 291). Cette séquence onirique anéantit l'espace patriarcal, fait voler en éclats les interdits multiples et revendique la dune de la Barga comme l'espace du désir assumé, transformé et abouti.

\section{La mer dans Mes Hommes}

Lors de sa première découverte de la mer, Mokeddem y voit une alternative créative à l'enfermement du désert. En ce sens, elle semble paraphraser et féminiser le vers de Baudelaire qui ouvre cette revue 1 : "[Femme] libre, toujours, tu chériras la mer!":

J'ai découvert la mer seulement quand je suis arrivée à Oran, à l'université. J'aimais aller la regarder. Juste ça. La contempler. Longtemps. M'en rassasier. J'observais ses mouvements, ses humeurs. Puis je fermais les yeux, respirais ses bouffées d'iode, sa fraîcheur. Bercée par son chant, j'oubliais l'enfer du désert. [...] Les Algériens étaient encore un peuple qui tournait le dos à la mer. En partie, sans doute, à cause de tous les envahisseurs que les vagues avaient crachés sur leurs côtes depuis des millénaires. Ensuite la plage, ses ébats, ses "dépravations", étaient restés l'apanage des Français (Mokeddem, 2006: 156-157).

Certes, les Français ont envahi et colonisé l'Algérie, et effectivement, la prise d'Alger s'était bien effectuée par la mer comme l'évoque Assia Djebar dans L'Amour, la Fantasia:

Aube de ce 13 juin 1830, à l'instant précis où le jour éclate au-dessus de la conque profonde. Il est cinq heures du matin. Devant l'imposante flotte qui déchire l'horizon, la Ville Imprenable se dévoile, blancheur fantomatique, à travers un poudroiement de bleus et de gris mêlés [...]. La ville, paysage tout en dentelures et en couleurs

\footnotetext{
${ }^{1}$ Note de l'éditeur: l'auteur de cet article fait référence au vers de Baudelaire cité en épigraphe au texte de l'appel à contribution pour le numéro inaugural de Carnets.
} 
délicates, surgit dans un rôle d'Orientale immobilisée en son mystère (Djebar, 1985: 14).

Pourtant, cette "dépravation" importée et pratiquée par les Français qui vient de la mer et y retourne est parée de bien des attraits. N'est-elle pas séduite par un navigateur français?

je rencontre Alain, un homme venu de la mer. II a accosté de la mer en voilier. II est Français. II a pris la mer pour fuir un désespoir [...]. II a pris la mer pour survivre. La mer l'a restitué à la vie. II me raconte son errance en bateau, ponctue son récit de suggestions: "Je t'enlève? Je quitte le port. Tu m'attends sur une plage. Je t'embarque. Ni vu, ni connu. Finis, les tracas de ce pays!' (Mokeddem, 2006: 82)

D'une manière plus générale, la mer est liée pour elle à la sensualité, à la fois à la découverte de sensations qu'elle expérimente en mangeant, en buvant, en "dévorant" selon ses propres termes:

Saïd m'invite à dîner en bord de mer. II me conseille une langouste grillée, "excellente ici”. Je n'en ai jamais mangé. Je dévore le crustacé. Je trouve ça tellement bon que je m'en lèche les doigts. Ses yeux verts et les lumières de la mer ne sont pas étrangers à mon euphorie. J'ai bien échappé à toutes les noirceurs, les nausées, les rages du désert. Là-bas, je n'aurais pas pu me laisser aller à ce sentiment merveilleux pour un homme. L'amour me sauve d'abord de mes propres furies.

Je vois, je bois des rayons verts. Ses yeux. La mer. [...] La rumeur de la mer, ses lueurs resteront le creuset de nos divagations amoureuses. Ses embruns, ses saveurs, les yeux dans les yeux, la mer à ressac, le voyage de l'amour (Mokeddem, 2006: 70-71).

N'oublions pas que Saïd est Kabyle, c'est-à-dire qu'il est porteur d'une différence. D'autre part, la mention du "festin maritime" préfigure de manière à peine voilée la fête des sens et elle prend toute son intensité jubilatoire et libératrice quand on le rapproche de son passé d'anorexique alors qu'elle vivait dans le désert. Elle ne sera sauvée de cette maladie que par le Docteur Shalles, qui, ayant l'intuition de sa vocation médicale, l'arrachera à l'anéantissement et à l'autodestruction. II lui demande de l'assister pour soigner les malades lors de ses tournées, et, dans le même temps, en l'accueillant chez lui, et en lui faisant découvrir des romans, des livres de poésie, de la musique classique et lyrique. Le Docteur Shalles et son épouse qui est sage-femme, apparaissent dans son récit autobiographique comme de véritables substituts parentaux, des tuteurs, en même temps que des passeurs 
culturels qui lui permettent d'échapper à ce qu'elle appelle "l'enfer du désert". Et la mer devient également un espace maternel, ou du moins, l'espace qu'il fallait traverser pour oublier sa propre mère, à moins qu'elle ne se réconcilie avec elle:

Je regarde le bateau au loin et dis à Jean-Louis: “Ça y est, j'ai traversé la mère!". II ne sait pas que je pense mère à la place de mer. Je ne me pose aucune question. Je n'ai aucune envie de m'embarrasser d'introspection. Je suis encore toute à mes images de la traversée. A cette glisse intemporelle sur la peau lisse de la Grande Bleue (Mokeddem, 2006: 158).

Les jeux de langage que Malika Mokeddem s'autorise dans ces lignes (mermère/glisse-lisse) sont suffisamment isolés pour qu'on y prête l'attention voulue. En effet, quelques pages plus loin après cette "traversée de la mère", son hymne à la mer prend des accents quasi-mystiques en jouant phonétiquement et sémantiquement sur la "dérive" entre les "deux rives":

Nous sommes devenus des gens du voyage. Ce mode de vie en voilier, je l'assimile au nomadisme de mes ancêtres. Ils avaient leurs pistes. J'ai un sillage que la mer efface aussitôt. A mon grand ravissement. J'aimerais faire durer la traversée. Savourer jusqu'à l'extase ces odyssées de bleus qui me bercent, me portent, me murmurent les rêves de la terre. Je suis Bleue en pleine mer. Je suis Dieu dans ce désert liquide. Ce cœur battant entre les deux rives de ma sensibilité. Je suis cette dérive (Mokeddem, 2006: 160).

\section{La mer dans N'zid}

Précisément, là est bien la question, la Méditerranée, dont l'étymologie nous rappelle qu'elle sépare deux terres, devient la ligne de partage pour Malika Mokeddem, comme le rappelle Denise Brahimi:

Du Sud-Oranais à Montpellier, comme chacun sait, il faut traverser la Méditerranée, qui figure dans ces livres comme un espace vide, un blanc entre les deux rives, dont les aspects et les particularités ne sont pas évoqués. C'est une mer qui sépare, et d'ailleurs c'est bien ainsi qui l'a voulu l'héroïne lorsque volontairement ou par la force des choses, elle a décidé de quitter son pays.

Le grand renversement de perspective et de point de vue, lorsqu'on arrive à N'zid, est que, dans cette histoire-là, tout est vu à partir de la mer sur laquelle et même dans laquelle se passe l'action (Brahimi, 2006: 135). 
Et si la mer est d'une certaine manière l'alpha et l'oméga de N'zid, il nous faut observer de quelle manière Malika Mokeddem, cette "voix singulière" comme l'a nommée Charles Bonn (2001: 130), utilise, travaille et transforme le motif maritime pour créer un espace fictionnel et existentiel à sa mesure. Najib Redouane rappelle encore la fonction de la Méditerranée comme lieu de naissance, de renaissance ou de continuité:

Dans N'zid, c'est la mer qui remplace le désert, devenant cet espace fondamental, conférant un côté sublime à tous ces lieux découverts des deux rives de la Méditerranée.

En fait, la Méditerranée apparaît intéressante en elle-même, mais aussi en tant que force mystérieuse et grandeur de l'infini qui, avec son flux et reflux, permet à Nora de poursuivre sa quête personnelle pour retrouver son identité et se retrouver avec ellemême. Elle est également un élément poétique reflétant les sentiments de la narratrice, en parvenant à esquisser son sort tragique, sa grande douleur et sa profonde déchirure (Redouane, 2003: 30).

Le titre même de l'œuvre N'zid en arabe (qui signifie "je continue" et aussi "je nais") nous pose question. En effet, il n'est pas courant que Malika Mokeddem utilise des termes arabes dans ses romans et quand elle le fait, c'est presque toujours avec une charge négative, pour dénoncer une réalité qui lui semble aliénante. Ce fait est particulièrement flagrant dans son roman L'Interdite (Mokeddem, 1993) où le personnage principal est une femme médecin qui revient au désert pour aider les villageois de son ksar. Les femmes qui la consultent lui font part de leur souffrance et de leurs symptômes:

II y a quelque chose qui me donne des coups de couteau ici et là [...]. Quand tout, en arabe algérien koulchi est douloureux, il s'agit de la koulchite, une pathologie féminine très commune et très connue ici. La koulchite, symptomatique des séismes et de l'angoisse au féminin (Mokeddem, 1993: 125).

La "koulchite" est un néologisme que notre auteure a créé et qui véhicule une expérience négative. Elle y fait d'ailleurs allusion dans N'zid (Mokeddem, 2001: 142). Pourtant, il n'y a rien de tel dans l'emploi du verbe employé à la première personne, et qui est lourd d'une promesse, celle d'une naissance (renaissance pour Nora) ou d'une continuité (poursuivre son existence, brutalement et mystérieusement coupée en deux par un traumatisme dont on sait seulement qu'elle "a perdu connaissance en mer").

La lecture de N'zid nous permet ainsi de percevoir la Méditerranée comme l'élément centripète du texte qui attire à lui tous les motifs et tous les éléments configurant 
l'interrogation profonde du roman: (re)naître? Continuer? Et, en filigrane, la question fondamentale: disparaître?

Alors qu'elle se réveille sur son bateau à la dérive, Nora perçoit la mer comme un élément bienfaisant, doté de caractéristiques physiques humaines:

La vue de la mer l'apaise. Elle ne lui est pas seulement familière. Elle est un immense cœur au rythme duquel bat le sien. En la regardant, elle rêve encore d'elle. Elle fait partie d'elle, patrie matrice. Flux des exils. Sang bleu du globe entre ses terres d'exode (Mokeddem, 2001: 25, c'est nous qui soulignons).

Mais Nora est devenue complètement amnésique en oubliant jusqu'à son nom, sa nationalité, sa profession et sa situation familiale. La Méditerranée apparaît à ce moment de façon tout à fait contradictoire comme son seul point d'ancrage alors que son bateau est à la dérive. Denise Brahimi a une perception qui nous semble fort juste en évoquant la thématique spatiale dans N'zid en termes de lieu et de non-lieu: "L'aspiration profonde que manifeste le comportement de Nora est le désir de se situer dans un non-lieu, qui se trouve être à la fois le non-lieu flottant du bateau, toujours en train de se déplacer, et le non-lieu de la mer, elle aussi toujours en mouvement" (Brahimi, 2006: 136).

Arrêtons-nous cependant quelques instants sur ce personnage féminin dépourvu au début du roman de nom et de nationalité, en un mot, dépourvu d'identité. Elle est interrogée par divers hommes qui croisent sa route (Loïc, un navigateur qui la suit depuis une escale puis l'aide à diverses reprises, le médecin qu'elle consulte pour en savoir plus long sur son amnésie, les commerçants chez qui elle s'approvisionne), en un mot, par des hommes qui lui veulent du bien. Pourtant, ils la mettent en demeure de s'identifier par rapport à une terre ou à une patrie. Elle varie alors ses réponses et se déclare tour à tour Grecque, Libanaise ou originaire de tout autre pays du Bassin Méditerranéen. II est vrai qu'au moment où elle fournit ces réponses, elle a perdu la mémoire de son origine, mais son inventivité géographique est aussi certainement une manière de se rattacher à la mer Méditerranée qui devient de fait la matrice du monde.

Nombre de critiques ont vu dans N'zid un aspect fondamental de l'œuvre mokeddienne, celui de la déterritorialisation qui, selon Valérie Orlando, "assure une cassure avec les anciens repères et une liberté vis-à-vis des origines. [...] Le sujet déterritorialisé n'a pas de limite de perception, mais est libre d'explorer de nouvelles possibilités" (Orlando, 2000:105). Effectivement, ce faisant, Malika Mokeddem abolit les frontières et permet de relier les habitants de pays s'affrontant sans relâche en leur donnant une même origine: 
La mer est son incantation. Elle est sa sensualité quand elle lèche les recoins les plus intimes des rivages, son sortilège quand elle hante les yeux des guetteurs. [...] Elle est sa complice quand elle roule, court, embrasse, dans une même étreinte, Grèce et Turquie, Israël, Palestine et Liban, France et Algérie. Elle est son rêve en dérive entre des bras de terre, à la traverse des détroits et qui va s'unir, dans un concert de vents, au grand océan. Sa Méditerranée est une déesse scabreuse et rebelle que ni les marchands de haine, ni les sectaires n'ont réussi à fermer. Elle est le berceau où dorment, au chant de leurs sirènes, les naufragés esseulés, ceux des causes perdues, les fuyards de Gibraltar et bien des illusions de vivants (Mokeddem, 2001: 68-69).

Ainsi, si la mer rassemble des frères traditionnellement ou historiquement ennemis dans la première partie du roman, elle va permettre peu à peu à Nora de reconstituer l'énigme parentale, la charade familiale, l'autre interrogation sur les origines, familiale, cette fois: "Mon père tailleur de pierres et de courants m'a donné la mer entre deux langues. De l'autre rive, la mère, elle, a mis ma vie entre elle et moi" (Mokeddem, 2001: 149).

Et peu à peu, elle redécouvre l'histoire de son père irlandais et de sa mère algérienne; cette alliance du Nord et du Sud semble a priori tout à fait inattendue chez Malika Mokeddem, si on replace cette œuvre après les cinq premiers romans écartelés entre l'Algérie et la France. C'est en cela que l'expression que Trudy Agar-Mendousse utilise pour évoquer N'zid, en parlant d'une "écriture d'apaisement" (Agar-Mendousse, 2006: 110) nous semble tout à fait justifiée.

On pourrait en effet comparer N'zid à une série d'énigmes que Nora résout peu à peu en ouvrant métaphoriquement une série de portes qui, à leur tour, ouvrent sur d'autres portes. II est possible que l'image de poupées gigognes pour évoquer la recherche de son identité soit peut-être plus fidèle à la réalité. Najib Redouane préfère, quant à lui, parler d' "odyssée intérieure" (Redouane, 2003: 31), reprenant l'idée d'une quête labyrinthique où tout est aimanté par la Méditerranée qui ordonne tous les motifs de l'œuvre.

Avant de parvenir à redonner à ses géniteurs noms et origines, grâce, d'ailleurs à une autre déracinée, Zana, femme de harki vivant en France, Nora reçoit de la mer une invitation, une stimulation à la création. II s'agit de manière plus précise, de peindre le portrait du père, tout en s'étonnant plus tard de l'absence de celui de la mère:

Elle regarde tour à tour la mer et le portrait de son père. Le besoin de peindre, celui de se jeter à l'eau, la regagnent, aussi indissociables qu'irrépressibles, dans la même intranquillité. Elle saisit pinceaux et fusains sans idée aucune de ce que ses mains vont produire (Mokeddem, 2001: 71). 
La mer bout et fume en lessivant le monde. Le ciel est tendu comme un linge amidonné. Le vent forcit, marmonne et grogne dans les voiles, joue les ratés de violon avec les haubans [...]. Une évidence occultée depuis deux jours, fait irruption dans son esprit. Elle la formule avec calme:

- Pas dessiné la mère... pourquoi? (Mokeddem, 2001: 101)

Signalons à ce propos et sans nous y attarder davantage, car cela mériterait un plus large développement, que la thématique maternelle est particulièrement ambiguë dans l'imaginaire mokeddien. A plus d'une reprise, elle souligne dans diverses entrevues, dans les portraits qui lui sont consacrés et dans nombre de ses romans combien les filles souffrent de la préférence clairement affichée des mères pour leurs fils, et des graves conséquences que ce manque imprime durablement aux petites filles devenues femmes. Ainsi, lisons-nous dans son premier roman Les Hommes qui marchent:

Saâdia était de l'avis de tous, une enfant splendide mais très mélancolique. "L'amour d'une mère, c'est la lumière du destin que l'enfant reçoit avec son lait. C'est ce manque qui dévore le regard de la pauvre Saâdia. C'est lui qui lui donne cette sombre avidité. Une faim qui restera à jamais inassouvie" (Mokeddem, 1990: 41-42).

En tirant le fil parental, la mère, puis le père, apparaît encore un thème récurrent dans N'zid, c'est celui de l'exil. Comme les habitants de la rive sud de la Méditerranée essayant d'en gagner la rive nord, le père de Nora a dû, lui aussi, s'exiler et elle prend soin d'indiquer qu'il s'agit de la Grande Famine d'Irlande de 1845 à 1849. Y répondent en écho les paroles de Malika Mokeddem dans un dialogue avec Najib Redouane:

L'exil n'est pas par rapport à un territoire, c'est par rapport à une tribu et à partir du moment où cette tribu était devenue étouffante, j'étais devenue l'étrangère par rapport à cette tribu. Mais j'étais en même temps délivrée de toute la pesanteur des tabous et des interdits, et je suis allée vers des horizons ouverts (Redouane, 2003: 295).

Elle recouvre peu à peu les éléments de son identité diluée dans la mer qui en a effacé toutes les traces (ne peut-on d'ailleurs pas voir dans le nom du bateau de Nora qui change, passant de "la Tramontane" à "L'Aimée" une sorte de "palimpseste naval" qui renforce la complexité des origines?).

Une autre énigme qui taraude Nora au long de la narration, c'est l'identité, encore et toujours - to be or not to be, that is the question - de ce mystérieux J., personnage masculin: est-ce Jean Rolland, mystérieusement disparu en Algérie, apparemment aux mains des islamistes, ou Jamil dont la musique donne son titre au roman? 
Avant de parvenir à déchiffrer l'énigme de l'ami et de l'amant, Nora scrute et observe la mer et y voit reflétées ses propres sensations, émotions ou préoccupations. De manière quasi constante, il existe une correspondance entre le paysage intérieur et le paysage extérieur; Bachelard parlerait de l'espace intérieur du "dedans" et de l'espace ouvert du "dehors":

Sous l'aile des voiles, Nora dort. Puis, elle se réveille, jette un œil autour du bateau. Aucun danger. Juste la mer blafarde comme une insomnie, sans visage, sans rivage. Silence sculpté par le vent. Crêtes de mémoire sur lames d'oubli. Même amputée. La lune masque encore les étoiles. Les plus lumineuses pointent à peine, gelées sous un ciel de papier (Mokeddem, 2001: 145).

Pourtant, l'acmé de son émotion s'incarne quand l'évocation de la mer amène celle du désert:

Curieusement elle pressent que le danger est ailleurs. Elle ignore où. Le souvenir d'un cauchemar de la veille lui revient tout à coup à l'esprit. Elle naviguait sur une mer déchaînée. Des lames croisées menaçaient de disloquer le bateau. De gros nuages crevaient le ciel. Les vents tournants de l'orage soufflaient, soulevant de monstrueuses tornades d'eau. Peu à peu, toute cette fureur liquide a commencé à devenir rouge, un rouge sang. La tempête s'est muée en vent de sable. La mer s'est coagulée en désert. Au moment où une avalanche de dunes s'abattait sur elle, un épouvantable râle d'asphyxiée l'a réveillée (Mokeddem, 2001: 65).

Et Jamil, dont elle reconstitue patiemment l'histoire, est la figure qui parvient à synthétiser tous les traits la définissant: ne vient-il pas, lui aussi, du désert algérien? Musicien, n'est-il pas, lui aussi créateur, et enfin, n'incarne-t-il pas la notion postmoderne de la fusion et du métissage en redisant à Nora qu'étant "issue de trois terres, elle était au-delà de toute terre, dans une liberté plus grande, celle des mers, des airs, et de la création?" (Mokeddem, 2001: 207).

Le récit de leur rencontre est à la fois la pierre d'angle du récit et le point névralgique de l'œuvre dans la mesure où toutes les pièces du puzzle s'assemblent enfin pour donner la clé du mystère:

Soudain, dans la rumeur des vagues, c'est un autre morceau de luth qu'entend Nora. Un conciliabule crépusculaire des infinis qui parle dans le vent. Nora se tourne, cherche des yeux. Juché sur la falaise, un homme basané, presque noir, fait corps avec son instrument. II s'arrête. La regarde: 
- N'zid?

- “N'zid?”: “Je continue?", et aussi: “Je nais.” (Mokeddem, 2001: 160)

Eperdue de gratitude, elle acquiesce d'un signe de la tête. Elle voit le visage de Jamil. Elle voit son corps courbé par l'étreinte du luth, vibrant à sa plainte. II joue au bord de la mer comme dans son désert. [...] La voix de Jamil proteste, envahit la mer, rauque et chaude. Sa voix devient la mer (Mokeddem, 2001: 161).

Maintenant, il sait que les sables sont une traversée dont on a tenté de l'exclure. Maintenant, il l'entreprend chaque jour, des quatre coins du globe. Bercé par la mer, il retrouve ses espaces, recompose toutes leurs sonorités, leurs sensualités. Maintenant, la mer est son autre désert (Mokeddem, 2001: 163).

Denise Brahimi en se posant la question “N'zid, quelle naissance?” montre que pour Nora, naître, ou plutôt renaître, c'est assumer que "le poids des morts, ou en tout cas, la fidélité aux morts qu'on a aimés fait partie de la seule identité véritable, avec ce que l'on pourrait appeler la fidélité à soi-même" (Brahimi, 2006: 143).

En qualifiant plus haut la rencontre avec Jamil de point névralgique du récit, on laisse entendre qu'elle porte en germe sa propre disparition, mort de l'aimé qui clôt le récit et pousse une fois encore Nora vers une autre mer, dans une fidélité aux morts libératrice et transformatrice:

Dans son murmure étouffé, Nora entend les premiers râles d'une tempête de sable et le sanglot d'un luth brisé:

"Il faut publier l'album sur Jamil'

- Qu'est-ce que tu veux faire?

- N'zid.

- Tu?... Tu veux aller en Algérie?

- Pas tout de suite. Les tombes peuvent attendre. J'ai une autre mer à traverser. Je veux emmener Tramontane dans le Gulf Stream, à Galway. J'irai chercher le luth de Jamil plus tard. Je me rendrai au désert lorsque le silence sera revenu là (Mokeddem, 2001: 214).

Après avoir parcouru avec Malika Mokeddem les différents espaces de l'écriture, les lieux opposés, désert ou mer Méditerranée, nous avons pris conscience de la richesse de sa perception du monde. II nous semble particulièrement important de souligner à quel point sa problématique est résolument moderne. De fait, en s'affranchissant des frontières ou, du moins, en cherchant à lutter contre la peur, les tabous et les poids des conventions, elle 
parvient à incarner un être qui se définit par sa propre identité, forgée au gré des échanges, des voyages, mais en aucun cas, tributaire du seul lieu de naissance. 


\section{Bibliographie}

Agar-Medousse, Trudy (2006). Violence et créativité de l'écriture algérienne au féminin. Paris: Editions L'Harmattan, pp. 105-186.

BONN, Charles (2001). "Littérature maghrébine francophone, horizon 2001: de quelques avatars de la perception 'groupale' d'une littérature qui a cessé d'être 'émergente'”. In: Beïda Chikhi (ed.), Vives Lettres, $\mathrm{n}^{\circ} 11$, Numéro spécial $\left(1^{\text {er }}\right.$ semestre 2001): Passerelles francophones. Pour un nouvel espace d'interprétation, volume II: Afrique et Antilles. Strasbourg: Université Marc Bloch, pp. 117132.

Boustani, Carmen et Jouve, Edmond (dir.) (2006). Des femmes et de l'écriture. Le bassin méditerrannéen. Paris: Editions Karthala.

BraHIMI, Denise (2006). "N'zid: quelle naissance?". In: Carmen Boustani et Edmond Jouve (dir.) (2006). Des femmes et de l'écriture. Le bassin méditerrannéen. Paris: Editions Karthala, pp. 133143.

Bueno Alonso, Josefina (2004). "Femme, identité, écriture dans les textes francophones du Maghreb". In: Thélène. Revista Complutense de Estudios Franceses, № 19, pp. 7-20.

CHIKHI, Beïda (2001). "Passerelles francophones". In: Beïda Chikhi (ed.), Vives Lettres, no11, Numéro spécial $\left(1^{\mathrm{er}}\right.$ semestre 2001).

DJEBAR, Assia (1985). L'Amour, la Fantasia. Paris: Albin Michel.

HELM, Yolande Aline (dir.) (2000). Malika Mokeddem: envers et contre tout. Paris: L'Harmattan.

LEJEUNE, Philippe (1975). Le pacte autobiographique. Paris: Seuil.

MiCHEL-MANSOUR, Thérèse (2003). "Symbolique du désert dans les œuvres de Malika Mokeddem". In: Najib Redouane, Yvette Bénayoun-Szmidt, Robert Elbaz (dir.). Malika Mokeddem. Paris: Editions L'Harmattan, Col. Autour des écrivains maghrébins, pp. 171-183.

MoKEDDEM, Malika (1990). Les Hommes qui marchent. Paris: Ramsay.

MOKEDDEM, Malika (1993). L'Interdite. Paris: Grasset.

MOKEDDEM, Malika (1995). Des rêves et des assassins. Paris: Grasset.

MOKEDDEM, Malika (2001). N'zid. Paris: Seuil.

MOKEDDEM, Malika (2006). Mes Hommes. Paris: Grasset.

ORLANDO, Valérie (2000). "Ecriture d'un autre lieu: la déterritorialisation des nouveaux rôles féminins dans L'Interdite". In: Yolande Aline Helm (dir.) Malika Mokeddem: envers et contre tout. Paris: L'Harmattan, pp. 105-115.

Redouane, Najib, Benayoun-Szmidt, Yvette, Elbaz, Robert (dir.) (2003). Malika Mokeddem. Paris: Editions L'Harmattan, Col. Autour des écrivains maghrébins.

RedouAne, Najib (2003). "A la rencontre de Malika Mokeddem". In: Najib Redouane, Yvette BénayounSzmidt, Robert Elbaz (dir.). Malika Mokeddem. Paris: Editions L'Harmattan, Col. Autour des écrivains maghrébins, pp. 17-39.

SegarRA, Marta (1997). Leur pesant de poudre: romancières francophones du Maghreb. Paris: Editions L'Harmattan. 